\title{
Monitoring of the application of laminating adhesives to polyurethane foam by near infrared chemical imaging
}

\author{
G. Mirschel, ${ }^{a}$ O. Daikos, ${ }^{a}$ C. Steckert ${ }^{\mathrm{b}}$ and T. Scherzer ${ }^{\mathrm{a} *}$ \\ aLeibniz Institute of Surface Engineering (IOM), Chemical Department, Permoserstr. 15, D-04318 Leipzig, Germany. E-mail: tom.scherzer@iom-leipzig.de
}

bLLA Instruments GmbH, Justus-von-Liebig-Str. 9/11, D-12489 Berlin, Germany

\begin{abstract}
Near infrared chemical imaging was used for the quantitative determination of the application weight of hot-melt adhesive layers laminated to polyetherbased polyurethane (PUR) foam as well as for monitoring of its spatial distribution across the surface of the foam. Adhesive layers were applied as melt-spun fibrous webs of a thermoplastic polyester with application weights between about $20 \mathrm{~g} \mathrm{~m}^{-2}$ and $130 \mathrm{~g} \mathrm{~m}^{-2}$. In spite of the coarsely porous surface structure of the foam, RMSEP was found to be $3.8 \mathrm{~g} \mathrm{~m}^{-2}$ only. Chemical images of the adhesive layers revealed that a minimum of two adhesive webs corresponding to an application weight of about $40 \mathrm{~g} \mathrm{~m}^{-2}$ are required for the formation of a continuous layer without holes. Average application weights derived from the chemical images have shown an excellent correlation with gravimetric reference values.
\end{abstract}

\section{Introduction}

Spectroscopic methods belong to the most powerful and most widely used approaches in quality and process control, since (i) they can be operated in a contact-free mode without any impact on the material studied, (ii) they can provide a broad range of information without significant time delay and (iii) they can achieve very high precision when they are combined with chemometric approaches for the quantitative evaluation of the spectral data. In particular, near infrared (NIR) reflection spectroscopy has proven to be a powerful tool for in-line process control during the last two decades. ${ }^{1}$ The analysis of the material under investigation is carried out in a spot with a size of a few millimetres only, corresponding to the diameter of the probe beam of the spectrometer or in a small strip in the case of moving materials such as paper, polymer webs, textiles, materials on a conveyor etc. For many technical applications, this is considered to be sufficient. However, in some applications the spatial distribution of the monitored parameter is essential, in particular if the homogeneity of the material is of interest. For such problems,
NIR chemical imaging using large hyperspectral cameras offers immense potential for a large-scale quantitative in-line analytics.

In the past, NIR hyperspectral imaging has been used in numerous investigations focused on the characterisation of a wide variety of foods and agricultural products. ${ }^{2,3}$ In contrast, only very few applications in chemistry have been reported. Apart from the well-known spectroscopic classification of synthetic polymers for plastic waste sorting, 4,5 only some investigations in the field of polymer processing have been carried out so far. One of the earliest studies was dealing with the curing of a phenol formaldehyde resin used as bonding material for sandpapers. ${ }^{6}$ Other investigations were focused on quality control in polymer extrusion processes, ${ }^{7}$ the crystallisation of polymers ${ }^{8}$ or monitoring the properties of natural rubber. 9

Textile fabrics, synthetic leather or mats of foam plastic provided with an adhesive layer for further processing are typical intermediate products, which are widely used for

\section{Correspondence}

T. Scherzer (tom.scherzer@iom-leipzig.de)

doi: $10.1255 /$ nir2017.163

Citation: G. Mirschel, O. Daikos, C. Steckert and T. Scherzer, "Monitoring

of the application of laminating adhesives to polyurethane foam by near

infrared chemical imaging", in Proc. $18^{\text {th }}$ Int. Conf. Near Infrared Spectrosc., Ed

by S.B. Engelsen, K.M. Sørensen and F. van den Berg. IM Publications Open,

Chichester, pp. 163-168 (2019). https://doi.org/10.1255/nir2017.163

\section{(c) 2019 The Authors}

This licence permits you to use, share, copy and redistribute the paper in any medium or any format provided that a full citation to the original paper is given.

ISBN: 978-1-906715-27-4 
interior design in the automotive industry and similar industries such as wagon construction etc. They are used for roof and door linings, rear panel shelfs, for padding, coverings or sound insulation. These materials may be either laminated to other layers such as fabrics or are directly adhered to any substrate. Laminating adhesives used for such applications are mostly thermoplastic aliphatic polyesters or polyamides, which melt at temperatures between about $110^{\circ} \mathrm{C}$ and $160^{\circ} \mathrm{C}$. Their application can be carried out as melt with an anilox roll, as powder with subsequent fusing or as melt-spun fibrous web, which is laminated with the web substrate. In order to achieve optimum adhesion properties as well as high-quality external surfaces after bonding, the adhesive has to be applied with high homogeneity.

Due to its irregular pore structure, the homogeneous application of adhesives to foam plastics is challenging. Lamination with area-measured adhesive webs has been proven to be one of the most promising methods of application. Nevertheless, inhomogeneities may result from tears, folds, holes, local fluctuation in the area density of the adhesive web or from penetration of the molten adhesive into the pores of the foam. Therefore, this study deals with the use of NIR chemical imaging for in-line monitoring of the thickness and the homogeneity of adhesive layers applied to foam plastics and its perspective application for process control.

\section{Experimental \\ NIR chemical imaging}

NIR spectra were recorded with a hyperspectral camera, KUSTA1.9MSI (LLA Instruments GmbH, Berlin, Germany), which is based on a thermoelectrically cooled InGaAs photodiode array with $192 \times 96$ pixels (spatial $\times$ spectral resolution). The sensor covers a spectral range from $1320 \mathrm{~nm}$ to $1900 \mathrm{~nm}$, which corresponds to a spectral resolution of $6 \mathrm{~nm} / \mathrm{pixel}$. The camera was mounted above a conveyor belt (Axmann Fördersysteme GmbH, Zwenkau, Germany) with a width of $500 \mathrm{~mm}$, the speed of which can be varied continuously from $2 \mathrm{~m} \mathrm{~min}^{-1}$ to $60 \mathrm{~m} \mathrm{~min}^{-1}$. The belt is equipped with a highly absorbing black polyurethane coating with a matted surface to limit contributions of the belt to the recorded spectra. In order to cover the complete width of the belt, the camera was equipped with a NIR objective $f=15 \mathrm{~mm} / 2.0$ (Specim,
Oulu, Finland). The resulting lateral resolution is about $2.6 \mathrm{~mm} /$ track.

Illumination of the samples on the belt was carried out with two parallel illumination units, each with four lamps in a row (120W, Osram). Spectra of the samples were recorded in reflection mode at a line speed of $10 \mathrm{~m} \mathrm{~min}^{-1}$, which corresponds to typical operating speeds in lamination technology. A more comprehensive description of experimental details can be found in Reference 10.

\section{Chemometrics}

Quantitative analysis of the spectral data was carried out by chemometric methods using the KustaSpec software package (LLA Instruments $\mathrm{GmbH}$ ). All chemometric models were based on the partial least squares (PLS) algorithm using reference data obtained by gravimetry. An internal validation set was used for the determination of the optimum number of eigenvectors. Models were optimised by application of different spectral pre-processing methods such as normalisation, baseline correction and derivatives in order to obtain maximum spectral information and minimum noise. In some cases, the spectral range considered by the PLS algorithm was limited (e.g. to exclude the influence of atmospheric humidity). For each model, parameters such as the root mean square error of prediction (RMSEP), the standard error of prediction (SEP), the bias and the coefficient of determination $\left(R^{2}\right)$ were calculated. Finally, the prediction capability of each model was characterised with an external validation set of independent samples.

The prediction of the application weights of unknown samples was carried out using the PLS model with the lowest RMSEP and the highest $R^{2}$. Chemical images were derived from the NIR spectra with the KustaBelt program (LLA Instruments $\mathrm{GmbH}$ ).

\section{Sample preparation}

White polyether-based polyurethane (PUR) foam $\left(\sim 280 \mathrm{~g} \mathrm{~m}^{-2}\right.$, sample size $\left.290 \times 210 \times 5 \mathrm{~mm}\right)$ was provided with an adhesive layer. The thermoplastic polyester adhesive was applied as melt-spun fibrous webs with a grammage of about 20-25 g m${ }^{-2}$ (Cepatex; CEPAT AG, Chur, Switzerland). Figure 1 shows an example of an adhesive web. Lamination was carried out at $150^{\circ} \mathrm{C}$ using a Meyer RMS Laminator (Herbert Meyer GmbH, Roetz, Germany). The application weight of the adhesive layer was varied by laminating one to seven webs to the foam. 


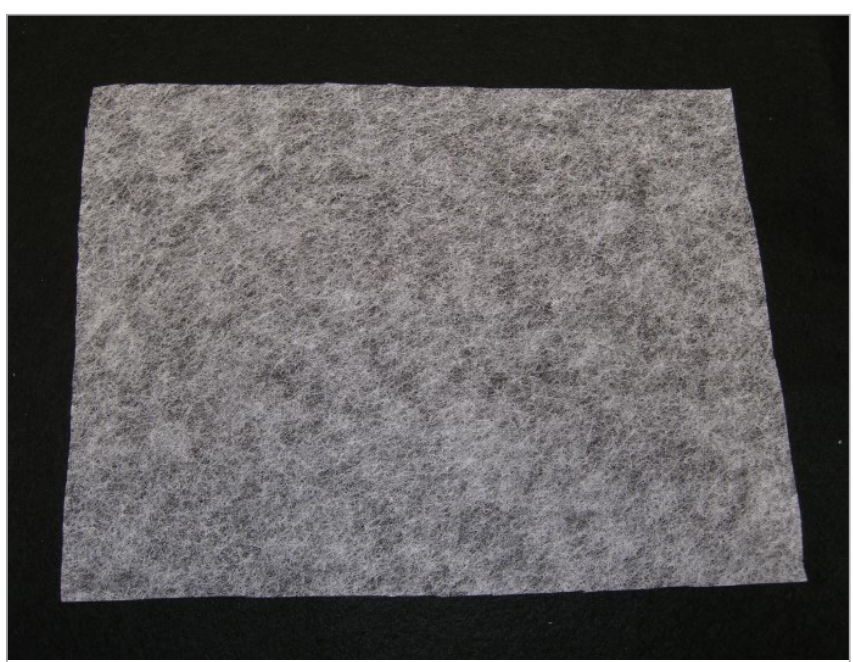

Figure 1. Polyester-based adhesive web.

The weights of foam samples and adhesive webs as well as the resulting weights of the laminates were determined with an analytical balance. Due to local fluctuation of the weight per area of all materials, a substantial variation of the site-specific weight of the laminates was observed.

\section{Results and discussion}

In order to vary the application weight of the adhesive on the PUR foam, different numbers of adhesive webs were laminated to foam substrates. In this way, the application weight was varied between about $20 \mathrm{~g} \mathrm{~m}^{-2}$ and $130 \mathrm{~g} \mathrm{~m}^{-2}$. Additionally, uncoated foam samples were included into the sample set as blanks. All samples were prepared in triplicate. Their actual application weight was determined by gravimetry. Moreover, NIR spectra of all samples were recorded during passage below the hyperspectral camera (Figure 2). The absorption of the aliphatic adhesive is clearly observable by means of the $2 \nu \mathrm{CH}$ overtones between $1650 \mathrm{~nm}$ and $1700 \mathrm{~nm}$ as well as by the slight increase of the combination band around $1410 \mathrm{~nm}$. For each sample, the data set comprised about 60,000 spectra. The spectra of two sample sets were used as calibration and validation set. A number of PLS calibration models with different spectral pre-treatments was built up. Finally, a model based on normalisation and application of the $1^{\text {st }}$ derivative and with a spectral range limited to the region from $1325 \mathrm{~nm}$ to $1841 \mathrm{~nm}$ was found to yield the lowest RMSEP and the highest $R^{2}$. The

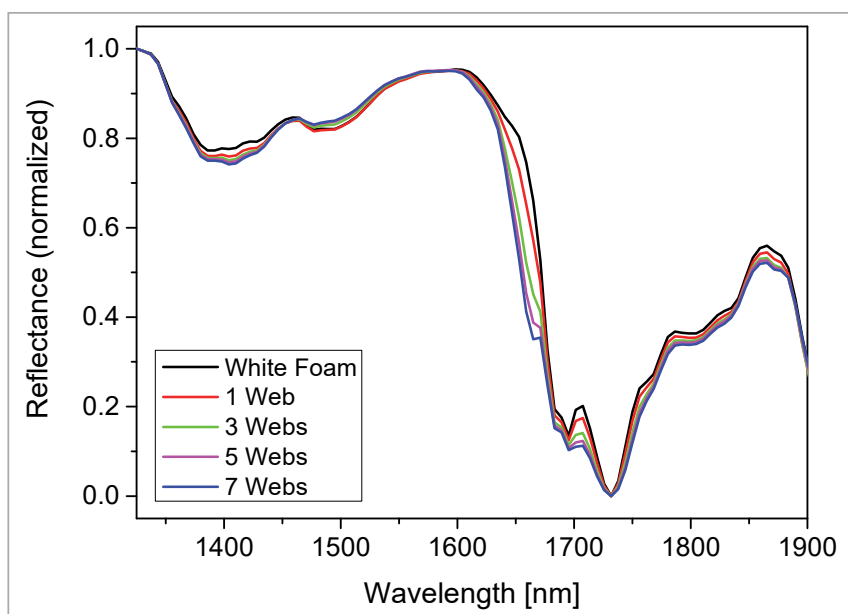

Figure 2. NIR spectra of aliphatic polyester layers with different thicknesses on polyether-based PUR foam.

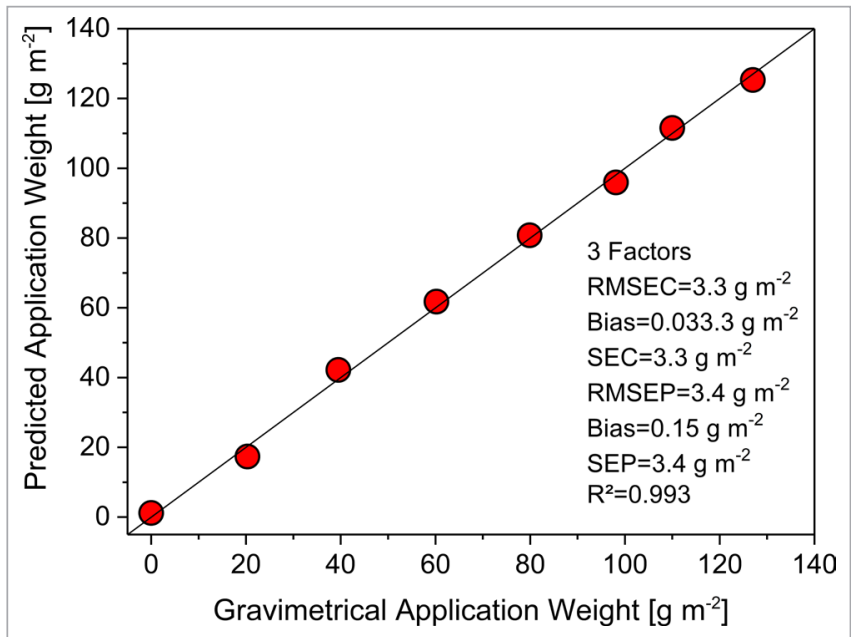

Figure 3. PLS calibration model for the application weight of an aliphatic polyester adhesive on $280 \mathrm{~g} \mathrm{~m}^{-2}$ white polyether-based PUR foam.

resulting calibration curve is shown in Figure 3. The most relevant parameters of the calibration model are given in this figure as well.

The third sample set mentioned above was used for the external validation of this calibration model. The application weight of the adhesive layer on white PUR foam was predicted from the NIR spectra. In order to be comparable with the corresponding gravimetric data, the vast set of individual predicted values across the surface of each sample was averaged. These averaged predicted values and the reference data are plotted against each other in Figure 4. It is obvious that the predicted values reflect the gravimetric data with rather high precision. 


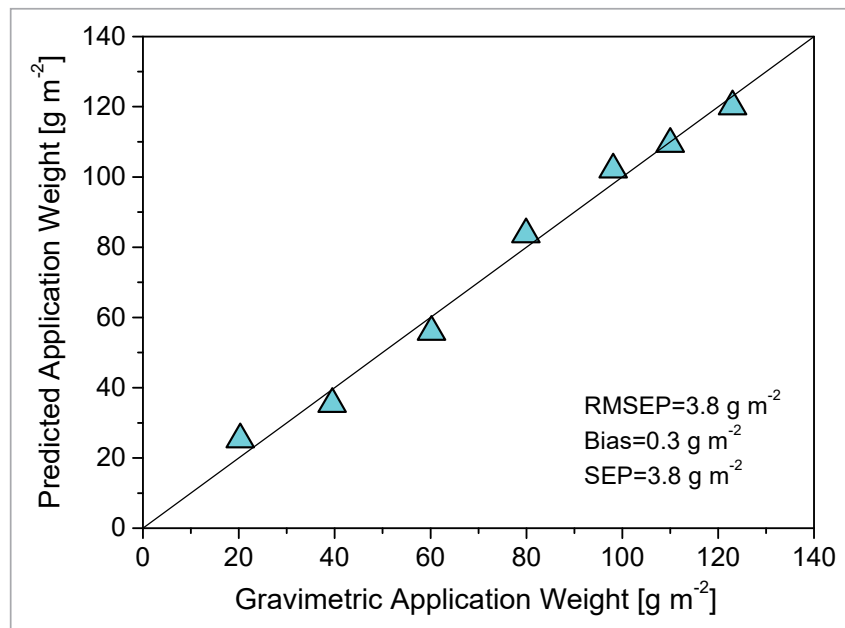

Figure 4. Prediction of the application weight of a polyester adhesive on white PUR foam using the PLS calibration model shown in Figure 3.

The prediction error was found to be $3.8 \mathrm{~g} \mathrm{~m}^{-2}$ only. This accuracy is regarded to be sufficient for the intended detection and visualisation of thickness variations.

After lamination at $150^{\circ} \mathrm{C}$, the adhesive forms a glossy transparent layer on the surface of the foam plastic. Therefore, it is difficult or even impossible to detect possible inhomogeneities visually, at least at higher application weights. This is the domain of hyperspectral imaging. Chemical images of the spatial distribution of the polyester adhesive on the surface of PUR foam were derived from the recorded NIR spectra using the above calibration model. Figure 5 shows three examples of adhesive layers with different application weights between about $20 \mathrm{~g} \mathrm{~m}^{-2}$ and $100 \mathrm{~g} \mathrm{~m}^{-2}$ applied to PUR foam.

It can be clearly seen from the chemical images, that the coating weight shows significant local variation if only one adhesive web had been laminated to the PUR foam (a similar, but somewhat weaker, effect is also apparent for the sample with two layers of web). This inhomogeneous distribution results from the coarsely porous structure at the surface of the PUR foam. During the melting process, the adhesive partly flows into the open bubbles at the surface of the foam leading to an uneven distribution. With increasing application weight, this effect becomes less significant for the formation of a continuous adhesive layer. Consequently, after application of minimum three webs to the foam the distribution of the adhesive appears to be much more homogeneous. Obviously, the open bubbles are closed now by the first

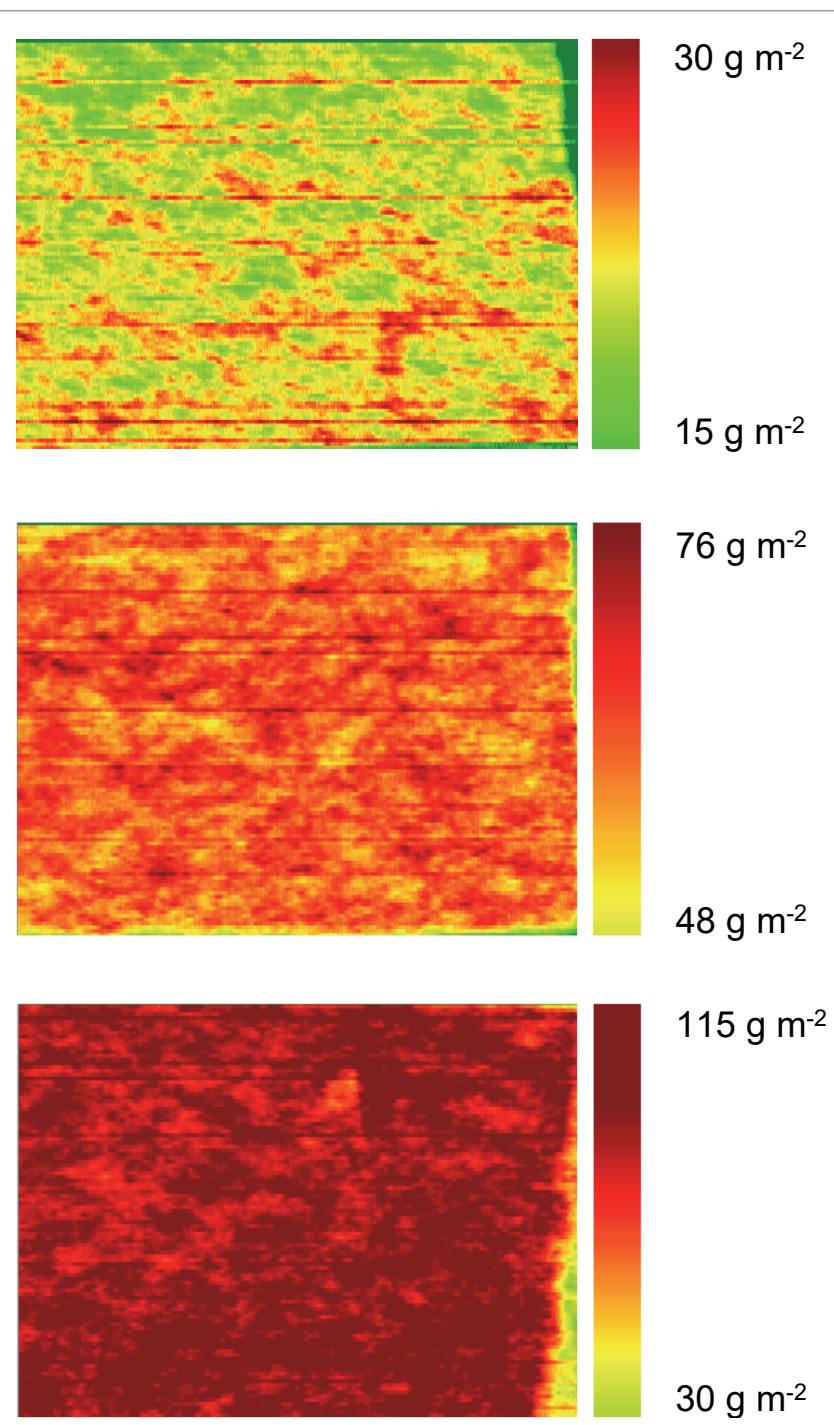

Figure 5. Chemical images of the distribution of a polyester adhesive on the surface of white PUR foam. Samples were prepared with one, three or five adhesive webs (from top to bottom). The corresponding gravimetric application weights were $20.3,60.2$ and $98.1 \mathrm{~g} \mathrm{~m}^{-2}$, respectively.

adhesive layers forming the basis for the development of a continuous coating. The weight distributions apparent in the various chemical images correspond to the visual impression of the samples: whereas samples with one or two adhesive webs clearly reveal surface defects such as remaining open pores, the surfaces of the samples provided with thicker adhesive layers appear to be rather homogeneous.

The average application weight of the adhesive on the foam can be determined from the chemical images by averaging the predicted individual values of all spectra 
Table 1. Comparison of the gravimetric application weights of polyester adhesive layers on white PUR foam with predictions from the chemical images obtained by integration across the complete surface.

\begin{tabular}{|c|c|c|}
\hline $\begin{array}{c}\text { Gravimetric } \\
\text { coating } \\
\text { weight }\left(\mathrm{g} \mathrm{m}^{-2}\right)\end{array}$ & $\begin{array}{c}\text { Predicted } \\
\text { coating weight } \\
\left(\mathrm{g} \mathrm{m}^{-2}\right)\end{array}$ & $\begin{array}{c}\text { Difference } \\
\left(\mathrm{g} \mathrm{m}^{-2}\right)\end{array}$ \\
\hline 20.3 & 19.6 & -0.7 \\
\hline 39.5 & 40.5 & 1.0 \\
\hline 60.2 & 62.0 & 1.8 \\
\hline 79.9 & 81.8 & 1.9 \\
\hline 98.1 & 99.5 & 1.4 \\
\hline 110.1 & 109.5 & -0.6 \\
\hline 123.2 & 124.1 & 0.9 \\
\hline
\end{tabular}

across the complete surface of each sample. These values may be used for direct comparison with the gravimetric application weights in order to evaluate the precision of the predictions. Table 1 summarises both values for the coated PUR foam samples of the third (independent) series. The close correlation between both data sets is apparent, even although the samples with lower application weights of the adhesive (i.e. with one or two adhesive webs) show rather poor homogeneity. These results clearly demonstrate the potential of NIR chemical imaging for space-resolved quantitative determination of the thickness or the application weight of rather thin polymer layers.

After the analytical verification that spectral imaging in general and the developed chemometric model in particular are able to determine the application weight of an adhesive on coarsely porous PUR foam with high precision, the method was used to characterise some samples from a real technical production process, where adhesive is applied to foam plastics at a working width of several metres. The materials used in this process, that is the polyether-based PUR foam as well as the thermoplastic polyester adhesive, are the same as those studied in the previous lab-scale investigations in this work. Samples were cut from the web with dimensions of $190 \times 140 \mathrm{~mm}$. Investigations by NIR chemical imaging were carried out on the conveyor system described above and at the same line speed. Quantitative data were obtained by means of the PLS calibration model. The chemical images of two production samples are shown in Figure 6. The specified application weight of the adhesive was $75 \mathrm{~g} \mathrm{~m}^{-2}$.

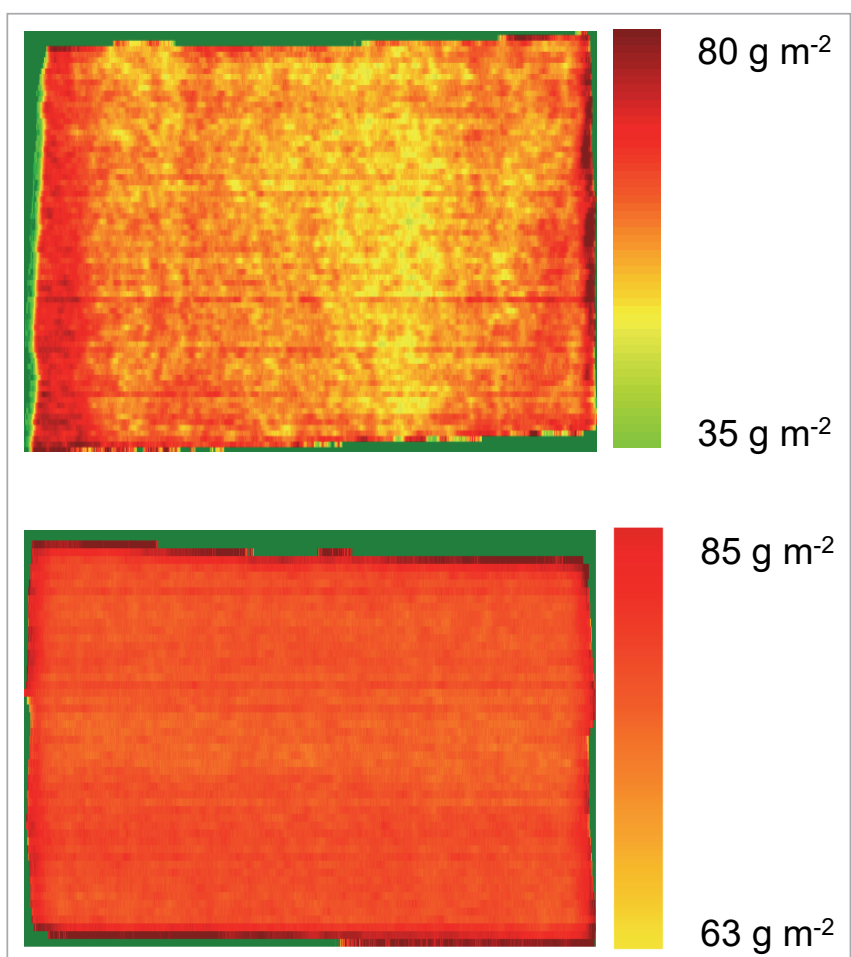

Figure 6. Chemical images of the distribution of a polyester adhesive on the surface of white PUR foam. Samples were taken from a production process. The specified application weight of the adhesive was $75 \mathrm{~g} \mathrm{~m}^{-2}$.

It is apparent that the quality of both samples differs considerably with respect to the homogeneity of the application of the adhesive to the foam substrate. In the case of the first sample, the distribution of the adhesive is rather inhomogeneous. In particular, there are large areas in the central regions where its application weight is considerably too low. Integration over all predicted values across the chemical image of the sample resulted in an average application weight of $56.4 \mathrm{~g} \mathrm{~m}^{-2}$, which is significantly lower than the specified value. In contrast, the distribution of the adhesive on the surface of the second sample appears to be much more homogeneous. In fact, averaging across the sample yielded an application weight of $72.8 \mathrm{~g} \mathrm{~m}^{-2}$.

\section{Conclusions}

In this work it was demonstrated that NIR chemical imaging can be used for the quantitative determination of the application weight of thin transparent adhesive layers on polymer materials with an irregular surface structure 
such as foam plastics. Moreover, the spatial distribution of the thickness of the adhesive across the surface of the foam was monitored. Adhesive layers with application weights between about $20 \mathrm{~g} \mathrm{~m}^{-2}$ and $130 \mathrm{~g} \mathrm{~m}^{-2}$ were applied by lamination of melt-spun fibrous webs made of a thermoplastic polyester to white polyether-based PUR foam. In spite of the coarsely porous surface structure of the foam, which hampers the formation of a homogeneous coating, the prediction error (RMSEP) was found to be $3.8 \mathrm{~g} \mathrm{~m}^{-2}$ only. Chemical images of the various foam/ adhesive laminates revealed that minimum two adhesive webs corresponding to an application weight of about $40 \mathrm{~g} \mathrm{~m}^{-2}$ are required to form continuous layers without holes caused by open bubbles of the foam. Average application weights derived from the chemical images of each sample by integration over all individual predicted values across their complete surface have shown an excellent correlation with gravimetric reference values. The outcome of this study clearly demonstrates the exceeding potential of NIR chemical imaging for spaceresolved quantitative characterisation of comparatively thin polymer layers. Similar investigations have been also carried out with dark foam plastics as well as for the hidden adhesive layers inside three-layer textile laminates intended for automotive applications. ${ }^{10}$ Moreover, the application weight of pressure-sensitive adhesives on textile fabrics was studied.

\section{Acknowledgements}

The authors gratefully acknowledge financial support of this project by the Arbeitsgemeinschaft industrieller Forschungsvereinigungen "Otto von Guericke" (AiF, Berlin, Germany; grant no. KF 2074011 KM3).

\section{References}

1. D.A. Burns and E.W. Ciurczak (Eds), Handbook of Near-Infrared Analysis, $3^{\text {rd }}$ Edn. CRC Press, Boca Raton (2008).

2. A.A. Gowen, C.P. O'Donnell, P.J. Cullen, G. Downey and J.M. Frias, "Hyperspectral imaging - an emerging process analytical tool for food quality and safety control", Trends Food Sci. Technol. 18, 590-598 (2007). https://doi.org/10.1016/j.tifs.2007.06.001
3. M. Manley, "Near-infrared spectroscopy and hyperspectral imaging: non-destructive analysis of biological materials", Chem. Soc. Rev. 43, 8200-8214 (2014). https://doi.org/10.1039/c4cs00062e

4. A. Kulcke, C. Gurschler, G. Spock, R. Leitner and M. Kraft, "On-line classification of synthetic polymers using near infrared spectral imaging", J. Near Infrared Spectrosc. 11, 71-81 (2003). https://doi. org/10.1255/jnirs.355

5. S. Serranti, A. Gargiulo and G. Bonifazi, "Hyperspectral imaging for process and quality control in recycling plants of olefin flakes", J. Near Infrared Spectrosc. 20, 573-581 (2012). https://doi. org/10.1255/jnirs.1016

6. T. Lillhonga, J. Dahlbacka and P. Geladi, "Hyperspectral near infrared image analysis of a phenol formaldehyde resin curing reaction", J. Near Infrared Spectrosc. 20, 559-572 (2012). https://doi. org/10.1255/1015

7. R. Gosselin, D. Rodrigue and C. Duchesne, "Potential of hyperspectral imaging for quality control of polymer blend films", Ind. Eng. Chem. Res. 48, 30333042 (2009). https://doi.org/10.1021/ie800997x

8. D. Ishikawa, T. Nishii, F. Mizuno, H. Sato, S.G. Kazarian and Y. Ozaki, "Potential of a newly developed highspeed near-infrared (NIR) camera (Compovision) in polymer industrial analyses: monitoring crystallinity and crystal evolution of polylactic acid (PLA) and concentration of PLA in PLA/poly-(R)-3-hydroxybutyrate (PHB) blends", Appl. Spectrosc. 67, 14411446 (2013). https://doi.org/10.1366/13-07103

9. C.J. da Silva and C. Pasquini, "Comparing nearinfrared conventional diffuse reflectance spectroscopy and hyperspectral imaging for determination of the bulk properties of solid samples by multivariate regression: determination of Mooney viscosity and plasticity indices of natural rubber", Analyst 140, 512-522 (2015). https://doi.org/10.1039/ c4an00836g

10. G. Mirschel. O. Daikos, T. Scherzer and C. Steckert, "Near-infrared chemical imaging used for in-line analysis of inside adhesive layers in textile laminates", Anal. Chim. Acta 932, 69-79 (2016). https:// doi.org/10.1016/j.aca.2016.05.015 